\title{
Riesz transforms on the Hardy space associated with generalized Schrödinger operators
}

\section{Yixin Wang ${ }^{1}$ and Pengtao $\mathrm{Li}^{1 *}$}

"Correspondence: ptli@qdu.edu.cn

'College of Mathematics and

Statistics, Qingdao University,

Qingdao, China

\begin{abstract}
Let $\mathcal{L}=-\Delta+\mu$ be a generalized Schrödinger operator, where the measure $\mu$ is a nonnegative Radon measure. In this paper, we establish the molecular characterization of the Hardy type space $H_{\mathcal{L}}^{1}\left(\mathbb{R}^{n}\right)$ associated with $\mathcal{L}$. As applications, we obtain the $H_{\mathcal{L}}^{1}$-boundedness of Riesz transforms and the imaginary power related to $\mathcal{L}$.

MSC: Primary 42B35; 47B38; 42B35

Keywords: Generalized Schrödinger operator; Hardy type space; Riesz transform; Molecular decomposition
\end{abstract}

\section{Introduction}

Consider the generalized Schrödinger operator

$$
\mathcal{L}=-\Delta+\mu \quad \text { in } \mathbb{R}^{n}, n \geq 3,
$$

where $\mu$ is a nonnegative Radon measure on $\mathbb{R}^{n}$. Throughout this paper we assume that $\mu$ satisfies the following conditions: there exist positive constants $C_{0}, C_{1}$, and $\delta$ such that

$$
\mu(B(x, r)) \leq C_{0}\left(\frac{r}{R}\right)^{n-2+\delta} \mu(B(x, R))
$$

and

$$
\mu(B(x, 2 r)) \leq C_{1}\left\{\mu(B(x, r))+r^{n-2}\right\}
$$

for all $x \in \mathbb{R}^{n}$ and $0<r<R$, where $B(x, r)$ denotes the open ball centered at $x$ with radius $r$. Condition (1.2) may be regarded as scale-invariant Kato-condition, and (1.3) says that the measure $\mu$ is doubling on balls satisfying $\mu(B(x, r)) \geq c r^{n-2}$.

Hardy spaces are widely used various fields of analysis and partial differential equations. Let $\Delta$ be the Laplace operator on $\mathbb{R}^{n}$. It is well known that $H^{1}\left(\mathbb{R}^{n}\right)$ can be characterized by the maximal function $\sup _{t>0}\left|e^{-t \Delta} f(x)\right|$. See Stein [14]. In a sense, $H^{1}\left(\mathbb{R}^{n}\right)$ can be seen as the Hardy space associated with the operator $-\Delta$. Let $\mathcal{L}$ be a general differential operator, such

(c) The Author(s) 2019. This article is distributed under the terms of the Creative Commons Attribution 4.0 International License (http://creativecommons.org/licenses/by/4.0/), which permits unrestricted use, distribution, and reproduction in any medium, provided you give appropriate credit to the original author(s) and the source, provide a link to the Creative Commons license, and indicate if changes were made. 
as second order elliptic self-adjoint operators in divergence form, degenerate Schrödinger operators with nonnegative potential, Schrödinger operators with nonnegative potential, and so on. In recent years, the Hardy spaces associated with $\mathcal{L}$ have become one of hot issues in harmonic analysis, see $[2,4-10]$ and the references therein.

Let $\mathcal{L}$ be a generalized Schrödinger operator. Denote by $\left\{T_{t}\right\}_{t>0}:=\left\{e^{-t \mathcal{L}}\right\}_{t>0}$ the heat semigroup generated by $-\mathcal{L}$. The kernel of $\left\{T_{t}\right\}$ is denoted by $K_{t}^{\mathcal{L}}(\cdot, \cdot)$, that is,

$$
T_{t} f(x)=\int_{\mathbb{R}^{n}} K_{t}^{\mathcal{L}}(x, y) f(y) d \mu(y) .
$$

The maximal function associated with $\left\{T_{t}\right\}$ is defined as

$$
\mathcal{M}_{\mathcal{L}}(f)(x):=\sup _{t>0}\left|e^{-t \mathcal{L}} f(x)\right| \in L^{1}\left(\mathbb{R}^{n}\right)
$$

In [15], Wu and Yan introduced the following Hardy type space associated with $\mathcal{L}$.

Definition 1.1 A Hardy type space $H_{\mathcal{L}}^{1}\left(\mathbb{R}^{n}\right)$ related to $\mathcal{L}$ is defined as the set of all functions in $f \in L^{1}\left(\mathbb{R}^{n}\right)$ satisfying $\mathcal{M}_{\mathcal{L}}(f) \in L^{1}\left(\mathbb{R}^{n}\right)$. The norm of $H_{\mathcal{L}}^{1}\left(\mathbb{R}^{n}\right)$ is defined as $\|f\|_{H_{\mathcal{L}}^{1}}:=$ $\left\|\mathcal{M}_{\mathcal{L}}(f)\right\|_{L^{1}}$

Let $\mathcal{L}=-\Delta . H_{\mathcal{L}}^{1}\left(\mathbb{R}^{n}\right)$ goes back to the classical Hardy space $H^{1}\left(\mathbb{R}^{n}\right)$. For a linear operator $T$, one of the methods to derive the $H^{1}$-boundedness is the so-called "atomic-molecular" method. In recent years, several authors used this method to investigate the boundedness on Hardy spaces associated with operators, see $[3,11,13]$. In Sect. 3.1, via a class of $(1, q)$ type atoms associated with $\mathcal{L}$, we obtain the corresponding atomic characterization of $H_{\mathcal{L}}^{1}\left(\mathbb{R}^{n}\right)$, see Sect. 3.1. Further, in Sect. 3.2, we introduce the $(p, q, \varepsilon)$-moleculars associated with $\mathcal{L}$ and establish the molecular decomposition of $H_{\mathcal{L}}^{1}\left(\mathbb{R}^{n}\right)$, see Theorem 3.6. In Sect. 4, let $R_{\mathcal{L}}$ and $\mathcal{L}^{i \gamma}$ denote the Riesz transforms and the imaginary power associated with $\mathcal{L}$, i.e.,

$$
\left\{\begin{array}{l}
R_{\mathcal{L}}:=\nabla(-\Delta+\mu)^{-1 / 2} \\
\mathcal{L}^{\gamma}:=(-\Delta+\mu)^{i \gamma}
\end{array}\right.
$$

By the aid of the regularities of the integral kernels, we can apply Theorems $3.3 \& 3.6$ to derive the $H_{\mathcal{L}}^{1}$-boundedness of $R_{\mathcal{L}}$ and $\mathcal{L}^{i \gamma}$, see Theorems $4.4 \& 4.6$, respectively.

Throughout this article, we will use $c$ and $C$ to denote the positive constants, which are independent of the main parameters and may be different at each occurrence. By $B_{1} \sim B_{2}$, we mean that there exists a constant $C>1$ such that $1 / C \leq B_{1} / B_{2} \leq C$.

\section{Preliminaries}

\subsection{Generalized Schrödinger operators}

Let $\mu$ be a Radon measure satisfying conditions (1.2) \& (1.3). The auxiliary function $m(x, \mu)$ is defined by

$$
\frac{1}{m(x, \mu)}=: \sup \left\{r>0: \frac{\mu(B(x, r))}{r^{n-2}} \leq C_{1}\right\} .
$$

We begin by recalling some basic properties of the function $m(x, \mu)$. 
Lemma 2.1 ([12, Proposition 1.8 \& Remark 1.9]) Suppose that $\mu$ satisfies (1.2) $\mathcal{E}$ (1.3). Then

(i) $0<m(x, \mu)<\infty$ for every $x \in \mathbb{R}^{n}$.

(ii) If $r=m(x, \mu)^{-1}$, then $r^{n-2} \leq \mu(B(x, r)) \leq C_{1} r^{n-2}$.

(iii) If $|x-y| \leq C m(x, \mu)^{-1}$, then $m(x, \mu) \approx m(y, \mu)$.

(iv) There exist constants $c, C>0$ such that, for $x, y \in \mathbb{R}^{n}$,

$$
\frac{c m(y, \mu)}{\{1+|x-y| m(y, \mu)\}^{k_{0} /\left(1+k_{0}\right)}} \leq m(x, \mu) \leq C m(y, \mu)\{1+|x-y| m(y, \mu)\}^{k_{0}}
$$

with $k_{0}=C_{2} / \delta>0$ and $C_{2}=\log _{2}\left(C_{1}+2^{n-2}\right)$.

With the modified Agmon metric $d s^{2}=m(x, \mu)\left\{d x_{1}^{2}+\cdots+d x_{n}^{2}\right\}$, the distance function $d(x, y, \mu)$ is given by

$$
d(x, y, \mu)=\inf _{\gamma} \int_{0}^{1} m(\gamma(\tau), \mu)\left|\gamma^{\prime}(\tau)\right| d \tau
$$

where $\gamma:[0,1] \rightarrow \mathbb{R}^{n}$ is absolutely continuous and $\gamma(0)=x, \gamma(1)=y$.

A parabolic-type distance function associated with $m(x, \mu)$ is defined by

$$
d_{\mu}(x, y, t)=\inf _{\gamma} \int_{0}^{1} m(\tilde{\gamma}(\tau), \mu) \max \left\{\left|(\tilde{\gamma})^{\prime}(\tau)\right|,\left|\left(\gamma_{n+1}\right)^{\prime}(\tau)\right|\right\} d \tau,
$$

where $\gamma(\tau)=\left(\gamma_{1}(\tau), \ldots, \gamma_{n}(\tau)\right)=\left(\tilde{\gamma}(\tau), \gamma_{n+1}(\tau)\right):[0,1] \rightarrow \mathbb{R}^{n} \times \mathbb{R}_{+}$is absolutely continuous with $\gamma(0)=(x, 0), \gamma(1)=(y, \sqrt{t})$.

Lemma 2.2 ([15, Lemma 2.2]) For the distance function $d(x, y, \mu)$ in (2.1), we have

(i) For every $x, y, z \in \mathbb{R}^{n}$,

$$
d(x, y, \mu) \leq d(x, z, \mu)+d(z, y, \mu) .
$$

(ii) There are two positive constants $c$ and $C$ such that, for any $x, y \in \mathbb{R}^{n}$,

$$
c\left\{\{1+|x-y| m(x, \mu)\}^{1 /\left(k_{0}+1\right)}-1\right\} \leq d(x, y, \mu) \leq C\{1+|x-y| m(x, \mu)\}^{k_{0}+1} .
$$

Lemma 2.3 ([15, Lemma 2.3]) For the distance function $d_{\mu}(x, y, t)$, there exist two positive constants $c$ and $C$ such that, for any $x, y \in \mathbb{R}^{n}, x \neq y$, and $t>0$,

$$
\left\{\begin{array}{l}
d_{\mu}(x, y, t) \geq c\left\{\{1+\max \{|x-y|, \sqrt{t}\} m(x, \mu)\}^{1 /\left(k_{0}+1\right)}-1\right\} \\
d_{\mu}(x, y, t) \leq C\{1+\max \{|x-y|, \sqrt{t}\} m(x, \mu)\}^{k_{0}+1}
\end{array}\right.
$$

It follows from (1.2), (1.3), and Lemma 2.1 that there exists a constant $C>0$ such that, for every $x \in \mathbb{R}^{n}$,

$$
\mu(B(x, r)) \leq \begin{cases}C(r m(x, \mu))^{\delta} r^{n-2}, & r<m(x, \mu)^{-1} ; \\ C(r m(x, \mu))^{C_{2}} m(x, \mu)^{2-n}, & r<m(x, \mu)^{-1},\end{cases}
$$

see $[15,(2.1)]$. The above estimate implies the following. 
Lemma 2.4 ([15, (2.2)]) For every nonnegative Schwarz function $\omega$,

$$
\int_{\mathbb{R}^{n}} t^{-n / 2} \omega\left(\frac{x-y}{\sqrt{t}}\right) d \mu(y) \leq \begin{cases}C t^{-1}(\sqrt{t} m(x, \mu))^{\delta}, & t<m(x, \mu)^{-2} \\ C t^{-1}(\sqrt{t} m(x, \mu))^{C_{2}-n+2}, & t \geq m(x, \mu)^{-2}\end{cases}
$$

\subsection{Function spaces associated with $\mathcal{L}$}

In order to characterize $H_{\mathcal{L}}^{1}\left(\mathbb{R}^{n}\right)$, Wu and Yan [15] introduced the following $H_{\mathcal{L}}^{1}$-atoms. For $j \in \mathbb{Z}$, define the sets $\mathcal{B}_{j}$ as

$$
\mathcal{B}_{j}=\left\{x: 2^{j / 2} \leq m(x, \mu)<2^{(j+1) / 2}\right\} .
$$

Since $0<m(x, \mu)<\infty$, we have $\mathbb{R}^{n}=\bigcup_{j \in \mathbb{Z}} \mathcal{B}_{j}$.

Definition 2.5 A function $a$ is a $(1, \infty)$-atom for $H_{\mathcal{L}}^{1}\left(\mathbb{R}^{n}\right)$ associated with a ball $B\left(x_{0}, r\right)$ if

(i) $\operatorname{supp} a \subset B\left(x_{0}, r\right)$;

(ii) $\|a\|_{L^{\infty}} \leq\left|B\left(x_{0}, r\right)\right|^{-1}$;

(iii) if $x_{0} \in \mathcal{B}_{j}$, then $r \leq 2^{1-j / 2}$;

(iv) if $x_{0} \in \mathcal{B}_{j}$ and $r \leq 2^{-1-j / 2}$, then $\int a(x) d x=0$.

The atomic norm of $H_{\mathcal{L}}^{1}\left(\mathbb{R}^{n}\right)$ is defined by $\|f\|_{\mathcal{L} \text {-atom }}:=\inf \left\{\sum_{j}\left|\lambda_{j}\right|\right\}$, where the infimum is taken over all decompositions $f=\sum_{j} \lambda_{j} a_{j}$, where $\left\{a_{j}\right\}$ is a sequence of $(1, \infty)$-atoms and $\left\{\lambda_{j}\right\}$ is a sequence of scalars.

One of the main results of [15] is the following proposition.

Proposition 2.6 ([15, Theorem 1.2]) Assume that $\mu$ is a nonnegative Radon measure on $\mathbb{R}^{n}$ satisfying (1.2) $\mathcal{E}(1.3)$ for some $\delta>0$. Then the norms $\|f\|_{H_{\mathcal{L}}^{1}}$ and $\|f\|_{\mathcal{L} \text {-atom }}$ are equivalent, that is, there exists a constant $C>0$ such that

$$
\frac{1}{C}\|f\|_{H_{\mathcal{L}}^{1}} \leq\|f\|_{H_{\mathcal{L}}^{1-\text { atom }}} \leq C\|f\|_{H_{\mathcal{L}}^{1}}
$$

At the end of this section, we state some regularity estimates for the kernel $K_{t}^{\mathcal{L}}(\cdot, \cdot)$.

Proposition 2.7 ([15, Lemma 3.7])

(i) There exist positive constants $C$ and $c$ depending only on $n$ and constants $C_{0}, C_{1}$ and $\delta$ in (1.2) \& (1.3) such that

$$
0 \leq K_{t}^{\mathcal{L}}(x, y) \leq C h_{t}(x-y) e^{-c d_{\mu}(x, y, t)}
$$

(ii) For every $0<\delta^{\prime}<\delta_{0}=\min \{\alpha, \delta, \nu\}$, there exists a constant $C$ such that, for every $N^{\prime}>0$, there exists a constant $C>0$ such that, for $|h|<\sqrt{t}$, we have

$$
\left|K_{t}^{\mathcal{L}}(x+h, y)-K_{t}^{\mathcal{L}}(x, y)\right| \leq C_{N^{\prime}}\left(\frac{|h|}{\sqrt{t}}\right)^{\delta^{\prime}} \frac{1}{t^{n / 2}} e^{-c|x-y|^{2} / t} \frac{C_{N}}{\{1+\sqrt{t} m(x, \mu)+\sqrt{t} m(y, \mu)\}^{N^{\prime}}} .
$$




\section{Molecular characterization of $H_{\mathcal{C}}^{1}\left(\mathbb{R}^{n}\right)$}

\subsection{The $(1, q)$-atom decomposition}

Now we introduce a new type of atoms.

Definition 3.1 A function $a$ is a $(1, q)$-atom of $H_{\mathcal{L}}^{1}\left(\mathbb{R}^{n}\right)$ if

(i) $\operatorname{supp} a \subset B\left(x_{0}, r\right)$;

(ii) $\|a\|_{q} \leq\left|B\left(x_{0}, r\right)\right|^{1 / q-1}$;

(iii) if $r \leq \rho\left(x_{0}\right)$, then $\int a(x) d x=0$.

Theorem 3.2 Any $(1, \infty)$-atom is a $(1, q)$-atom.

Proof In fact, by Hölder's inequality,

$$
\|a\|_{q} \leq\|a\|_{\infty}\left|B\left(x_{0}, r\right)\right|^{1 / q} \leq\left|B\left(x_{0}, r\right)\right|^{1 / q-1} .
$$

Theorem 3.3 Let $\mathcal{L}=-\Delta+\mu$ be a generalized Schrödinger operator, where $\mu \neq 0$ is a nonnegative Radon measure on $\mathbb{R}^{n}$ satisfying (1.2) \& (1.3) for some $\delta>0$. Then $f \in H_{\mathcal{L}}^{1}\left(\mathbb{R}^{n}\right)$ if and only if $f=\sum_{j} \lambda_{j} a_{j}$, where $\left\{a_{j}\right\}$ are $(1, q)$-atoms and $\left\{\lambda_{j}\right\}$ are scalars.

Proof Because an $(1, \infty)$-atom is also an $(1, q)$-type atom, we only need to prove that there exists a constant $c$ such that, for any $(1, q)$-atom $a,\left\|\mathcal{M}_{\mathcal{L}}(a)\right\|_{1} \leq c$. Suppose that $a$ is a $(1, q)$ atom supported in $B\left(x_{0}, r\right)$. We write $\left\|\mathcal{M}_{\mathcal{L}}(a)\right\|_{1} \leq I_{1}+I_{2}$, where

$$
\left\{\begin{array}{l}
I_{1}:=\int_{B\left(x_{0}, 4 r\right)}\left|\mathcal{M}_{\mathcal{L}} a(x)\right| d x \\
I_{2}:=\int_{B^{c}\left(x_{0}, 4 r\right)}\left|\mathcal{M}_{\mathcal{L}} a(x)\right| d x
\end{array}\right.
$$

By Hölder's inequality and the $L^{q}$-boundedness of $\mathcal{M}_{\mathcal{L}}$, we can get

$$
I_{1} \leq\left\|\mathcal{M}_{\mathcal{L}} a\right\|_{q}\left|B\left(x_{0}, r\right)\right|^{1-1 / q} \leq C\|a\|_{q}\left|B\left(x_{0}, r\right)\right|^{1-1 / q} \leq C
$$

The estimation of $I_{2}$ is divided into two cases.

Case 1: $1 / m\left(x_{0}, \mu\right) \leq r \leq 1 / 4 m\left(x_{0}, \mu\right)$. For this case, by (i) of Lemma 2.7, we have

$$
\begin{aligned}
\mathcal{M}_{\mathcal{L}}(a)(x) & \leq c \sup _{t>0} \int_{B\left(x_{0}, r\right)} t^{-n / 2} e^{-|x-y|^{2} / t}(1+m(x, \mu) \sqrt{t})^{-N}|a(y)| d y \\
& \leq c \sup _{t>0} \int_{B\left(x_{0}, r\right)} t^{-n / 2} e^{-|x-y|^{2} / t}\left(1+|x-y|^{2} / \sqrt{t}\right)^{-n-N}(1+m(x, \mu) \sqrt{t})^{-N}|a(y)| d y .
\end{aligned}
$$

If $y \in B\left(x_{0}, r\right)$ and $\left|x-x_{0}\right|>4 r$, then $\left|y-x_{0}\right| \leq\left|x-x_{0}\right| / 4$ and $|y-x| \geq 3\left|x-x_{0}\right| / 4$. We can apply Lemma 2.1 to obtain

$$
\begin{aligned}
\left|\mathcal{M}_{\mathcal{L}}(a)(x)\right| & \leq c \sup _{t>0} \frac{1}{t^{n / 2}} \int_{B\left(x_{0}, r\right)}\left(\left|x-x_{0}\right| / \sqrt{t}\right)^{-n-N}(m(x, \mu) \sqrt{t})^{-N}|a(y)| d y \\
& \leq c\left|x-x_{0}\right|^{-n-N}[m(x, \mu)]^{-N} \int_{B\left(x_{0}, r\right)}|a(y)| d y \\
& \leq c\left|x-x_{0}\right|^{-n-N /\left(k_{0}+1\right)}\left[m\left(x_{0}, \mu\right)\right]^{-N /\left(k_{0}+1\right)},
\end{aligned}
$$


which gives

$$
\begin{aligned}
\int_{\left|x-x_{0}\right|>4 r}\left|\mathcal{M}_{\mathcal{L}} a(x)\right| d x & \leq c \int_{\left|x-x_{0}\right|>4 r}\left|x-x_{0}\right|^{-n-N /\left(k_{0}+1\right)}\left[m\left(x_{0}, \mu\right)\right]^{-N /\left(k_{0}+1\right)} d x \\
& \leq c\left|x-x_{0}\right|^{-n-N /\left(k_{0}+1\right)} r^{-N /\left(k_{0}+1\right)} \leq C,
\end{aligned}
$$

where in the last inequality we have used the fact that $1 \leq r m\left(x_{0}, \mu\right) \leq 4$.

Case2: $r<1 / m\left(x_{0}, \mu\right)$. By Proposition 2.7 and the symmetry of $K_{t}^{\mathcal{L}}(\cdot, \cdot)$, we have

$$
\left|K_{t}^{\mathcal{L}}(x, y+h)-K_{t}^{\mathcal{L}}(x, y)\right| \leq C_{N}(|h| / \sqrt{t})^{\delta^{\prime}} t^{-n / 2} e^{-|x-y|^{2} / c t}\{1+\sqrt{t} m(x, \mu)+\sqrt{t} m(y, \mu)\}^{-N} .
$$

Notice that $\left|y-x_{0}\right|<r,\left|x-x_{0}\right|>4 r \Rightarrow|x-y| \geq 3\left|x-x_{0}\right| / 4$. By the canceling condition of $a$, we can get

$$
\begin{aligned}
\left|\mathcal{M}_{\mathcal{L}} a(x)\right| & \leq \sup _{t>0}\left|\int_{B\left(x_{0}, r\right)}\left[K_{t}^{\mathcal{L}}(x, y)-K_{t}^{\mathcal{L}}\left(x, x_{0}\right)\right] a(y) d y\right| \\
& \leq c \sup _{t>0} \int_{B\left(x_{0}, r\right)} t^{-n / 2} e^{-|x-y|^{2} / c t}\left(\left|y-x_{0}\right| / \sqrt{t}\right)^{\delta^{\prime}}|a(y)| d y \\
& \leq c \sup _{t>0}\left\{t^{-n / 2} \int_{B\left(x_{0}, r\right)}(1+|x-y| / \sqrt{t})^{-n-\delta^{\prime}}\left|y-x_{0}\right|^{\delta^{\prime}}\left|t^{\delta^{\prime} / 2}\right| a(y) \mid d y\right\} \\
& \leq c r^{\delta^{\prime}}\left|x-x_{0}\right|^{-n-\delta^{\prime}},
\end{aligned}
$$

which gives

$$
\int_{\left|x-x_{0}\right| \geq 4 r}\left|\mathcal{M}_{\mathcal{L}} a(x)\right| d x \leq c \int_{\left|x-x_{0}\right| \geq 4 r} r^{\delta^{\prime}}\left|x-x_{0}\right|^{-n-\delta^{\prime}} d x \leq C .
$$

\subsection{Molecular characterization of $H_{\mathcal{L}}^{1}\left(\mathbb{R}^{n}\right)$}

Now we introduce the molecular of $H_{\mathcal{L}}^{1}\left(\mathbb{R}^{n}\right)$.

Definition 3.4 Let $1 \leq q \leq \infty, \varepsilon>0, b=1-1 / q+\varepsilon$. An $L^{q}$-function $M$ is called a $(1, q, \varepsilon)$ molecular centered at $x_{0}$ if

(i) $|x|^{n b} M(x) \in L^{q}\left(\mathbb{R}^{n}\right)_{j}$;

(ii) $\|M\|_{q}^{\varepsilon / b}\left\|\left|x-x_{0}\right|^{n b} M(\cdot)\right\|_{q}^{1-\varepsilon / b} \leq 1$;

(iii) if $x_{0} \in B_{k}$ and $\|M\|_{q}^{\{n(1 / q-1)\}^{-1}} \leq m\left(x_{0}, \mu\right)^{-1}, \int M(x) d x=0$.

Lemma 3.5 If $a$ is a $(1, q)$-atom supported on $B\left(x_{0}, r\right)$, $a$ is also $a(1, q, \varepsilon)$-molecular centered at $x_{0}$.

Proof Recall that $\|a\|_{q} \leq\left|B\left(x_{0}, r\right)\right|^{1 / q-1}$. It is easy to see that

$$
\int_{\mathbb{R}^{n}}|| x-\left.\left.x_{0}\right|^{n b} a(x)\right|^{q} d x \leq\left|B\left(x_{0}, r\right)\right|^{b q+1-q},
$$

which indicates that $\left|\cdot-x_{0}\right|^{n b} a \in L^{q}\left(\mathbb{R}^{n}\right)$ with $\left\|\left|\cdot-x_{0}\right|^{n b} a\right\|_{q} \leq\left|B\left(x_{0}, r\right)\right|^{q}$. Moreover, for $b=1-1 / q+\varepsilon$,

$$
\|a\|_{q}^{\varepsilon / b}\left\|\left|\cdot-x_{0}\right|^{n b} a(\cdot)\right\|_{q}^{1-\varepsilon / b} \leq\left|B\left(x_{0}, r\right)\right|^{(1 / q-1)(\varepsilon / b)}\left|B\left(x_{0}, r\right)\right|^{\varepsilon(1-\varepsilon / b)} \leq 1 .
$$


We only need to verify the canceling condition, i.e., $\|a\|_{q}^{1 /\{n(1 / q-1)\}} \leq m\left(x_{0}, \mu\right)^{-1}$. Denote by $\omega_{n}$ the volume of the unit ball in $\mathbb{R}^{n}$. It is clear that $\omega_{n}>1$ and $\|a\|_{q} \leq \omega_{n}^{(1 / q-1)} r^{n(1 / q-1)} \leq$ $r^{n(1 / q-1)}$, equivalently,

$$
r \leq\|a\|_{q}^{1 /\{n(1 / q-1)\}} \leq m\left(x_{0}, \mu\right)^{-1} .
$$

By the canceling condition of $(1, q)$-atoms, we can see that $\int_{\mathbb{R}^{n}} a(x) d x=0$. So $a$ is a $(1, q, \varepsilon)$ molecular centered at $x_{0}$.

Theorem 3.6 Let $1 \leq q \leq \infty, \varepsilon>0, b=1-1 / q+\varepsilon$. Then $f \in H_{\mathcal{L}}^{1}\left(\mathbb{R}^{n}\right)$ if and only iff $=$ $\sum_{j} \lambda_{j} M_{j}$, where $\left\{M_{j}\right\}$ are $(1, q, \varepsilon)$-moleculars and $\left\{\lambda_{j}\right\}$ are scalars with $\inf \sum_{j}\left|\lambda_{j}\right| \sim\|f\|_{H_{\mathcal{L}}^{1}}$, where the infimum is taken over all decompositions.

Proof We have known that any $(1, q)$-type atom is also a $(1, q, \varepsilon)$-type molecular. By Theorem 3.3, if $f \in H_{\mathcal{L}}^{1}\left(\mathbb{R}^{n}\right)$, then there exist a sequence of $(1, q)$-type atoms $\left\{a_{j}\right\}$ and a sequence of scalars $\left\{\lambda_{j}\right\}$ such that $f=\sum_{j} \lambda_{j} a_{j}$. This means that $f$ can be represented as a linear combination of $(1, q, \varepsilon)$-moleculars. Conversely, we only need to verify that, for any $(1, q, \varepsilon)$-molecular, $\|M\|_{H_{\mathcal{L}}^{1}} \leq C$. For simplicity, denote

$$
N_{\mathcal{L}}(M)=:\|M\|_{q}^{\varepsilon / b}\left\|\left|\cdot-x_{0}\right|^{n b} M(\cdot)\right\|_{q}^{1-\varepsilon / b}
$$

Without loss of generality, we assume that $N_{\mathcal{L}}(M)=1$ and $q=2$. Write $\sigma=\|M\|_{2}^{1 /\{n(1 / 2-1)\}}$. Let

$$
\left\{\begin{array}{l}
E_{0}=\left\{x:\left|x-x_{0}\right| \leq \sigma\right\} ; \\
E_{k}=\left\{x: 2^{k-1} \sigma<\left|x-x_{0}\right| \leq 2^{k} \sigma\right\}, \quad k \in N ; \\
B_{k}=\left\{x:\left|x-x_{0}\right| \leq 2^{k} \sigma\right\}, \quad k=0,1,2, \ldots
\end{array}\right.
$$

Denote by $\psi_{k}$ the characteristic function $\chi_{E_{k}}(x)$ and write $M(x)=\sum_{k} M_{k}(x)$, where $M_{k}:=$ $M(x) \psi_{k}(x)$.

Case 1: $\sigma \leq 1 / m\left(x_{0}, \mu\right)$. Then $\|M\|_{2}^{1 /\{n(1 / 2-1)\}} \leq m\left(x_{0}, \mu\right)^{-1}$ and $\int_{\mathbb{R}^{n}} M(x) d x=0$. The proof is similar to the classical case, and we omit it.

Case 2: $\sigma>1 / m\left(x_{0}, \mu\right)$. For this case, $\left\|\left|\cdot-x_{0}\right|^{n(1 / 2+\varepsilon)} M(\cdot)\right\|_{2}^{1-\varepsilon / b}=\|M\|_{2}^{-\varepsilon / b}$. Denote by $\sigma$ the term $\|M\|_{2}^{1 /\{n(1 / 2-1)\}}$. Then $\left\|\left|\cdot-x_{0}\right|^{n(1 / 2+\varepsilon)} M(\cdot)\right\|_{2}=\sigma^{n \varepsilon}$ and

$$
\frac{1}{\left|B_{0}\right|} \int_{\mathbb{R}^{n}}\left|M_{0}(x)\right|^{2} d x \leq \frac{1}{\sigma^{n}}\|M\|_{2}^{2}=\frac{1}{\sigma^{2 n}}
$$

which implies that $\left\|M_{0}\right\|_{2} \leq\left|B_{0}\right|^{-1 / 2}$.

For the term $M_{k}$, we have

$$
\begin{aligned}
\frac{1}{\left|B_{k}\right|} \int_{\mathbb{R}^{n}}\left|M_{k}(x)\right|^{2} d x & \leq \frac{1}{\left(2^{k-1} \sigma\right)^{n}}\left\|\left|\cdot-x_{0}\right|^{n b} M_{k}(\cdot)\right\|_{2}^{2}\left(2^{k-1} \sigma\right)^{-n(1+2 \varepsilon)} \\
& \leq\left(2^{k-1} \sigma\right)^{-2 n-2 \varepsilon n} \sigma^{2 n \varepsilon} \\
& \leq C_{n, \varepsilon}\left(2^{k} \sigma\right)^{-2 n} 2^{-2 k \varepsilon}
\end{aligned}
$$


that is, $\|M\|_{2} \leq C\left|B_{k}\right|^{-1 / 2} 2^{-k \varepsilon n}$. Let $a_{k}(x)=\lambda_{k}^{-1} M_{k}(x), k=0,1,2, \ldots$, where $\lambda_{k}=2^{-2 k \varepsilon n}$ and $a_{k}, k \in \mathbb{Z}_{+}$, are $(1,2)$-atoms. Hence $M(x)=\sum_{k} \lambda_{k} a_{k}(x)=\sum_{k} M_{k}(x)$ and $\sum_{k}\left|\lambda_{k}\right|=$ $C \sum_{k} 2^{-2 k \varepsilon n}<\infty$. Repeating the procedure of [3, Theorem 4], we can prove that $M \in$ $H_{\mathcal{L}}^{1}\left(\mathbb{R}^{n}\right)$. We omit the details, and this completes the proof of Theorem 3.6.

4 Operators on the Hardy type space $H_{\mathcal{L}}^{1}\left(\mathbb{R}^{n}\right)$

\subsection{The $H_{\mathcal{L}}^{1}$-boundedness of $\mathcal{L}^{i \gamma}$}

Let $q_{t}(\cdot, \cdot)$ denote the kernel of $e^{-t \mathcal{L}}-e^{-t(-\Delta)}$. We have

$$
q_{t}(x, y)=h_{t}(x-y)-K_{t}^{\mathcal{L}}(x-y)=\int_{0}^{t} \int_{\mathbb{R}^{n}} K_{s}^{\mathcal{L}}(x, t) h_{t-s}(z-y) d \mu(z) d s .
$$

The following estimate was obtained by Wu and Yan [15].

Lemma 4.1 ([15, Lemma 3.6])

(i) There exist constants $C$ and $c$ such that, for every $x, y \in \mathbb{R}^{n}$ and $t>0$,

$$
q_{t}(x, y) \leq \begin{cases}C(\sqrt{t} m(x, \mu))^{\delta} t^{-n / 2} e^{-|x-y|^{2} / c t}, & \sqrt{t} \leq m(x, \mu)^{-1} \\ C(\sqrt{t} m(y, \mu))^{\delta} t^{-n / 2} e^{-|x-y|^{2} / c t}, & \sqrt{t} \leq m(y, \mu)^{-1} \\ h_{t}(x-y), & \text { elsewhere. }\end{cases}
$$

(ii) For every $0<\delta^{\prime}<\min \{1, \delta\}$ and $C>0$, there exist constants $C^{\prime}$ and $c$ such that, for every $h, x, y \in \mathbb{R}^{n},|h| \leq|x-y| / 4,|h| \leq \operatorname{Cm}(y, \mu)^{-1}$, we have

$$
\left|q_{t}(x, y+h)-q_{t}(x, y)\right| \leq C^{\prime}(|h| m(x, \mu))^{\delta^{\prime}} t^{-n / 2} e^{-|x-y|^{2} / c t} .
$$

By the functional calculus, we can see that the kernel of $(-\Delta)^{i \gamma}-\mathcal{L}^{i \gamma}$ can be expressed as

$$
g(x, y):=\int_{0}^{\infty} t^{-i \gamma} q_{t}(x, y) \frac{d t}{t} .
$$

Lemma 4.2 Let $\mathcal{L}=-\Delta+\mu$ be a generalized Schrödinger operator, where $\mu \neq 0$ is a nonnegative Radon measure on $\mathbb{R}^{n}$ satisfying (1.2) \& (1.3) for some $\delta>0$.

(i) If $y \in B\left(x_{0}, r\right)$, then

$$
|g(x, y)| \leq C m\left(x_{0}, \mu\right)^{\delta}|x-y|^{\delta-n} .
$$

(ii) There exists $0<\delta^{\prime}<\delta$ such that

$$
\left|g(x, y)-g\left(x, x_{0}\right)\right| \leq C|x-y|^{-n}\left(\left|y-x_{0}\right| m(x, \mu)\right)^{\delta^{\prime}} .
$$

Proof (i). In fact, we can deduce (4.2) from Lemma 4.1. Precisely,

Case 1: $\sqrt{t} \leq 1 / m(y, \mu)$. Because $y \in B$, then $\left|y-x_{0}\right|<r<1 / m\left(x_{0}, \mu\right), m(y, \mu) \sim m\left(x_{0}, \mu\right)$. By (i) of Lemma 4.1, we can get

$$
|g(x, y)| \leq C m\left(x_{0}, \mu\right)^{\delta} \int_{0}^{\infty} t^{-n / 2+\delta / 2-1} e^{-|x-y|^{2} / c t} d t \leq C m\left(x_{0}, \mu\right)^{\delta}|x-y|^{\delta-n} .
$$


Case 2: $\sqrt{t}>1 / m(y, \mu)$. For this case, $\sqrt{t} m(y, \mu)>1$. Using Lemma 4.1 again, we can deduce that

$$
|g(x, y)| \leq C \int_{0}^{\infty} t^{-n / 2} e^{-|x-y|^{2} / c t}(\sqrt{t} m(y, \mu))^{\delta} \frac{d t}{t} \leq C m\left(x_{0}, \mu\right)^{\delta}|x-y|^{\delta-n}
$$

(ii). It follows from (4.1) that

$$
\begin{aligned}
\left|g(x, y)-g\left(x, x_{0}\right)\right| & =\left|\int_{0}^{\infty} t^{-i \gamma} q_{t}(x, y) d t / t-\int_{0}^{\infty} t^{-i \gamma} q_{t}\left(x, x_{0}\right) \frac{d t}{t}\right| \\
& \leq \int_{0}^{\infty} t^{-i \gamma}\left|q_{t}(x, y)-q_{t}\left(x, x_{0}\right)\right| \frac{d t}{t} .
\end{aligned}
$$

By (ii) of Lemma 4.1 and a direct computation, we get

$$
\begin{aligned}
\left|g(x, y)-g\left(x, x_{0}\right)\right| & \leq\left|\int_{0}^{\infty} t^{-i \gamma}\left[\left|y-x_{0}\right| m(x, \mu)\right]^{\delta^{\prime}} t^{-n / 2} e^{-c|x-y|}\right|^{2} / t \frac{d t}{t} \mid \\
& \leq C\left[\left|y-x_{0}\right| m(x, \mu)\right]^{\delta^{\prime}} \int_{0}^{\infty} t^{-n / 2} e^{-c|x-y|^{2} / t} \frac{d t}{t} \\
& \leq C|x-y|^{-n}\left[\left|y-x_{0}\right| m(x, \mu)\right]^{\delta^{\prime}} .
\end{aligned}
$$

This completes the proof of Lemma 4.2.

We recall that an operator $T$ taking $\mathcal{C}^{\infty}\left(\mathbb{R}^{n}\right)$ into $L_{\text {loc }}^{1}\left(\mathbb{R}^{n}\right)$ is called a Calderón-Zygmund operator if

(a) $T$ extends to a bounded operator on $L^{2}\left(\mathbb{R}^{n}, d x\right)$;

(b) there exists a kernel $K$ such that, for every $f \in L_{c}^{1}\left(\mathbb{R}^{n}, d x\right)$,

$$
T f(x)=\int_{\mathbb{R}^{n}} K(x, y) f(y) d y \quad \text { a.e. on }\{\operatorname{supp} f\}^{c} ;
$$

(c) the kernel $K$ satisfies

$$
\left\{\begin{array}{l}
|K(x, y)| \leq c /|x-y|^{n} \\
|K(x+h, y)-K(x, y)| \leq c|h|^{\delta} /|x-y|^{n+\delta} \\
|K(x, y+h)-K(x, y)| \leq c|h|^{\delta} /|x-y|^{n+\delta}
\end{array}\right.
$$

In [12], Shen proved the following result.

Theorem 4.3 Let $\mathcal{L}=-\Delta+\mu$ be a generalized Schrödinger operator, where $\mu \neq 0$ is a nonnegative Radon measure on $\mathbb{R}^{n}$ satisfying (1.2) $\mathcal{E}$ (1.3) for some $\delta>0$. Then, for $\gamma \in \mathbb{R}^{n}$, $\mathcal{L}^{i \gamma}$ is a Calderón-Zygmund operator.

Now we prove the $H_{\mathcal{L}}^{1}$-boundedness of $\mathcal{L}^{i \gamma}$.

Theorem 4.4 Let $\mu$ be a nonnegative Radon measure in $\mathbb{R}^{n}, n \geq 3$. Suppose that $\mu$ satisfies conditions (1.2) $\mathcal{E}$ (1.3) for some $\delta>0$. Then, for $\gamma \in \mathbb{R}^{n}, \mathcal{L}^{i \gamma}$ is bounded on $H_{\mathcal{L}}^{1}\left(\mathbb{R}^{n}\right)$. 
Proof We only need to prove that, for any $(1, \infty)$ atom $a, \mathcal{L}^{i \gamma}(a)$ is a $(1, q, \varepsilon)$-molecular and $\left\|\mathcal{L}^{i \gamma}(a)\right\|_{H_{\mathcal{L}}^{1}} \leq C$. Let $a$ be a $(1, \infty)$ atom supported on $B\left(x_{0}, r\right)$. Then $\|a\|_{\infty} \leq 1 /\left|B\left(x_{0}, r\right)\right|$. If $r<m\left(x_{0}, \mu\right)^{-1}, \int a(x) d x=0$. Set $B^{\sharp}=B\left(x_{0}, 2 / m\left(x_{0}, \mu\right)\right)$ and $B^{*}=B\left(x_{0}, 2 r\right)$. We divide the proof into three parts.

Part I: $\mathcal{L}^{i \gamma} a \in L^{q}\left(\mathbb{R}^{n}\right) \&|x|^{n b} L^{i \gamma}(a) \in L^{q}\left(\mathbb{R}^{n}\right)$.

$$
\begin{aligned}
\left\||x|^{n b} \mathcal{L}^{i \gamma} a\right\|_{q} & \leq\left\|x_{B^{*}}|\cdot|^{n b} \mathcal{L}^{i \gamma} a\right\|_{q}+\left\|x_{\left(B^{*}\right)^{c}}|\cdot|^{n b} \mathcal{L}^{i \gamma} a\right\|_{q} \\
& =\left(\int_{B^{*}}|x|^{q n b}\left|\mathcal{L}^{i \gamma}(a)\right|^{q} d x\right)^{1 / q}+\left(\int_{\left(B^{*}\right) c}|x|^{q n b}\left|\mathcal{L}^{i \gamma}(a)\right|^{q} d x\right)^{1 / q} \\
& \leq\left(r+\left|x_{0}\right|\right)^{n b}\left\|\mathcal{L}^{i \gamma} a\right\|_{q}+\left[\int_{\left(B^{*}\right)^{c}}|x|^{q n b}\left|\int_{\mathbb{R}^{n}} K_{\gamma}^{\mathcal{L}}(x, y) a(y) d y\right|^{q} d x\right]^{1 / q} .
\end{aligned}
$$

By the $L^{q}$-boundedness of $\mathcal{L}^{i \gamma}$ and Minkowski's inequality, $\left\||x|^{n b} \mathcal{L}^{i \gamma} a\right\|_{q} \leq S_{1}+S_{2}$, where

$$
\left\{\begin{array}{l}
S_{1}:=\left(r+\left|x_{0}\right|\right)^{n b}\|a\|_{q} \\
S_{2}:=\int_{B\left(x_{0}, r\right)}|a(y)|\left[\int_{\left(B^{*}\right)^{c}}|x|^{q n b}\left|K_{\gamma}^{\mathcal{L}}(x, y)\right|^{q} d x\right]^{1 / q}
\end{array}\right.
$$

Because $\|a\|_{q} \leq\left|B\left(x_{0}, r\right)\right|^{1 / q-1}$, then

$$
S_{1}=\left(r+\left|x_{0}\right|\right)^{n b}\|a\|_{q} \leq\left(r+\left|x_{0}\right|\right)^{n b} r^{n(1 / q-1)} .
$$

For the term $S_{2}$, recall that

$$
K_{\gamma}^{\mathcal{L}}(x, y)=\int_{0}^{\infty} t^{-i \gamma} K_{t}^{\mathcal{L}}(x, y) \frac{d t}{t}
$$

Proposition 2.7 implies that

$$
\begin{aligned}
\left|K_{\gamma}^{\mathcal{L}}(x, y)\right| & \leq C_{N} t^{-n / 2} \frac{e^{-c|x-y|^{2} / t}}{\{1+|x-y|[m(x, \mu)+m(y, \mu)]\}^{N}} \frac{d t}{t} \\
& \leq \frac{C_{N}}{\{1+|x-y|[m(x, \mu)+m(y, \mu)]\}^{N}} \frac{1}{|x-y|^{n}},
\end{aligned}
$$

which gives

$$
S_{2} \leq C \int_{B}|a(y)|\left[\int_{\left(B^{*}\right)^{c}}|x|^{q n b} \frac{1}{\{1+|x-y|[m(x, \mu)+m(y, \mu)]\}^{q N}} \frac{d x}{|x-y|^{q^{n}}}\right]^{1 / q} d y .
$$

For $x \in B$ and $y \in\left(B^{*}\right)^{c}$, we can see that $|x-y| \geq\left|x-x_{0}\right| / 2$. Notice that for $y \in B\left(x_{0}, r\right)$, $\left|x_{0}-y\right|<r$ and

$$
m(y, \mu)^{N} \geq\left[\frac{c m\left(x_{0}, \mu\right)}{\left\{1+\left|x_{0}-y\right| m\left(x_{0}, \mu\right)\right\}^{k_{0} /\left(k_{0}+1\right)}}\right]^{N} .
$$

Then, via a direct computation, we have

$$
S_{2} \leq C \int_{B}|a(y)| \frac{1}{m(y, \mu)^{N}}\left\{\int_{\left(B^{*}\right)^{c}} \frac{|x|^{q n b} d x}{\left|x-x_{0}\right|^{(n+N) q}}\right\}^{1 / q} d y
$$




$$
\begin{aligned}
\leq & C \int_{B}|a(y)| \frac{1}{m(y, \mu)^{N}}\left\{\left[\int_{\left(B^{*}\right) c} \frac{1}{\left|x-x_{0}\right|^{(n+N) q-q n b}} d x\right]^{1 / q}\right. \\
& \left.+\left[\int_{\left(B^{*}\right)} \frac{\left|x_{0}\right|^{q n b}}{\left|x-x_{0}\right|^{(n+N) q}} d x\right]^{1 / q}\right\} d y \\
\leq & C \int_{B} \frac{|a(y)|}{m(y, \mu)^{N}}\left\{r^{n \varepsilon-N}+\left|x_{0}\right|^{n b} r^{-n-N+n / q}\right\} d y \\
\leq & C \frac{\left\{1+r m\left(x_{0}, \mu\right)\right\}^{k_{0} N /\left(k_{0}+1\right)}}{m\left(x_{0}, \mu\right)^{N}}\left\{r^{n \varepsilon-N}+\left|x_{0}\right|^{n b} r^{-n-N+n / q}\right\}\left(\int_{B}|a(y)| d y\right)<\infty .
\end{aligned}
$$

Part II: $N_{\mathcal{L}}\left(\mathcal{L}^{i \gamma} a\right)=\left\|\mathcal{L}^{i \gamma} a\right\|_{q}^{\varepsilon / b}\left\|\left|\cdot-x_{0}\right|^{n b} \mathcal{L}^{i \gamma} a\right\|_{q}^{1-\varepsilon / b} \leq C$.

Case I: $r \geq \rho\left(x_{0}\right)$. Because $x \in\left(B^{*}\right)^{c}$ and $y \in B$,

$$
\left\|\mathcal{L}^{i \gamma} a\right\|_{q} \leq\left(\int_{B^{*}}\left|\mathcal{L}^{i \gamma} a(y)\right|^{q} d y\right)^{1 / q}+\left(\int_{\left(B^{*}\right) c}\left|\mathcal{L}^{i \gamma} a(y)\right|^{q} d y\right)^{1 / q} .
$$

Because $\mathcal{L}^{i \gamma}$ is bounded on $L^{q}\left(\mathbb{R}^{n}\right), q>1$, then

$$
\left(\int_{B^{*}}\left|\mathcal{L}^{i \gamma} a(y)\right|^{q} d y\right)^{1 / q} \leq\left\|\mathcal{L}^{i \gamma} a\right\|_{q} \leq C\|a\|_{q} \leq|B|^{1 / q-1} .
$$

For $y \in B$ and $x \in\left(B^{*}\right)^{c},|x-y| \geq\left|x-x_{0}\right| / 2$. By Theorem 4.3 , we can get

$$
\begin{aligned}
\left(\int_{\left(B^{*}\right) c}\left|\mathcal{L}^{i \gamma} a(x)\right|^{q} d x\right)^{1 / q} & \leq \int_{B}|a(y)|\left(\int_{\left(B^{*}\right)^{c}}\left|K_{\gamma}^{\mathcal{L}}(x, y)\right|^{q} d x\right)^{1 / q} d y \\
& \leq \int_{B}|a(y)|\left(\int_{\left(B^{*}\right)^{c}}|x-y|^{-n q} d x\right)^{1 / q} d y \\
& \leq \int_{B}|a(y)| r^{n / q-n} d y \leq C r^{n / q-n}
\end{aligned}
$$

Because $q>1 \& r \geq \rho\left(x_{0}\right)$, the above estimates indicate that

$$
\left\|\mathcal{L}^{i \gamma} a(x)\right\|^{1 /\{n / q-n\}} \geq \rho\left(x_{0}\right)^{(n / q-n) /\{n / q-n\}}=\rho\left(x_{0}\right)
$$

which means that such $\mathcal{L}^{i \gamma}$ a need not satisfy the canceling condition.

On the other hand, we write $\left\|\left|\cdot-x_{0}\right|^{n b} \mathcal{L}^{i \gamma} a\right\|_{q} \leq I_{1}+I_{2}$, where

$$
\left\{\begin{array}{l}
I_{1}:=\left\|\chi_{B^{*}}\left|\cdot-x_{0}\right|^{n b} \mathcal{L}^{i \gamma} a\right\|_{q} ; \\
I_{2}:=\left\|\chi_{\left(B^{*}\right) c}\left|\cdot-x_{0}\right|^{n b} \mathcal{L}^{i \gamma} a\right\|_{q} .
\end{array}\right.
$$

For $I_{1}$, by the $L^{q}$-boundedness of $\mathcal{L}^{i \gamma}$ and the fact that $\varepsilon-b=1 / q-1$, we have

$$
I_{1} \leq C|B|^{b}\left\|\mathcal{L}^{i \gamma} a\right\|_{q} \leq C|B|^{\varepsilon} .
$$

For $I_{2}$, because

$$
\left|K_{\gamma}^{\mathcal{L}}(x, y)\right| \leq \frac{C_{N}}{\{1+|x-y|[m(x, \mu)+m(y, \mu)]\}^{N}} \frac{1}{|x-y|^{n}}
$$


we can use Lemma 2.1 and the fact that $r \geq 1 / m\left(x_{0}, \mu\right)$ to obtain

$$
\begin{aligned}
I_{2} & \leq C \int_{B} \frac{|a(y)|}{m(y, \mu)^{N}}\left(\int_{\left(B^{*}\right)^{c}} \frac{\left|x-x_{0}\right|^{n b q}}{\left|x-x_{0}\right|^{(n+N) q}} d x\right)^{1 / q} d y \\
& \leq C \int_{B} \frac{|a(y)|}{m(y, \mu)^{N}} r^{n b+n / q-n-N} d y \\
& \leq C \int_{B}|a(y)|\left\{\frac{1}{m\left(x_{0}, \mu\right)}+\left|y-x_{0}\right|\right\}^{N} r^{n b+n / q-n-N} d y \\
& \leq C \int_{B}|a(y)| r^{N} r^{n b+n / q-n-N} d y \\
& \leq C|B|^{\varepsilon} .
\end{aligned}
$$

The estimates for $I_{1}$ and $I_{2}$ imply that

$$
N_{\mathcal{L}}\left(\mathcal{L}^{i \gamma} a\right)=\left\|\mathcal{L}^{i \gamma} a\right\|_{q}^{\varepsilon / b}\left\|\left|\cdot-x_{0}\right| \mathcal{L}^{i \gamma} a\right\|_{q}^{1-\varepsilon / b} \leq C
$$

Case 2: $r<1 / m\left(x_{0}, \mu\right)$. For this case, the atom $a$ has the canceling property. There exists a positive integer $m$ such that $2^{-m-1} / m\left(x_{0}, \mu\right) \leq r<2^{-m} / m\left(x_{0}, \mu\right)$. Let $B^{\sharp}=B\left(x_{0}, 2 / m\left(x_{0}, \mu\right)\right)$ and $B^{*}=B\left(x_{0}, 2 r\right)$. We write

$$
\mathcal{L}^{i \gamma} a=\left(\mathcal{L}^{i \gamma}-(-\Delta)^{i \gamma}\right) a+(-\Delta)^{i \gamma} a
$$

We will prove that $\left(\mathcal{L}^{i \gamma}-(-\Delta)^{i \gamma}\right) a$ and $(-\Delta)^{i \gamma} a$ are both moleculars. For $r<1 / m\left(x_{0}, \mu\right)$, any $(1, q)$-atom is a classical atom. By Alverez-Milman [1], $(-\Delta)^{i \gamma} a$ is a $(1, q, \varepsilon)$-molecular. Hence, $(-\Delta)^{i \gamma} a$ is a molecular of $H_{\mathcal{L}}^{1}\left(\mathbb{R}^{n}\right)$. We write $\left\|\left(\mathcal{L}^{i \gamma}-(-\Delta)^{i \gamma}\right) a\right\|_{q} \leq I_{1}+I_{2}+I_{3}$, where

$$
\left\{\begin{array}{l}
I_{1}:=\left\|\left(\mathcal{L}^{i \gamma}-(-\Delta)^{i \gamma}\right) a \chi_{B^{*}}\right\|_{q} ; \\
I_{2}:=\left\|\left(\mathcal{L}^{i \gamma}-(-\Delta)^{i \gamma}\right) a \chi_{B^{\sharp} \backslash B^{*}}\right\|_{q} ; \\
I_{3}:=\left\|\left(\mathcal{L}^{i \gamma}-(-\Delta)^{i \gamma}\right) a \chi_{\left(B^{\sharp}\right)}\right\|_{q} .
\end{array}\right.
$$

We first estimate the term $I_{1}$. Because $\delta \in(0, n)$, then $n / q-n+\delta>0$. Estimate (4.2) implies that

$$
\begin{aligned}
I_{1} & \leq C \int_{B}\left(\int_{B^{*}}|g(x, y)|^{q} d x\right)^{1 / q}|a(y)| d y \\
& \leq C \int_{B}|a(y)| m\left(x_{0}, \mu\right)^{\delta}\left(\int_{B^{*}}|x-y|^{-q(n-\delta)} d x\right)^{1 / q} d y \\
& \leq C \int_{B}|a(y)| m\left(x_{0}, \mu\right)^{\delta} r^{(n-q n+q \delta) / q} d y \\
& \leq C m\left(x_{0}, \mu\right)^{\delta}\left[2^{m} m\left(x_{0}, \mu\right)\right]^{-n / q+n-\delta} \\
& \leq C m\left(x_{0}, \mu\right)^{n-n / q} .
\end{aligned}
$$


Now we deal with $I_{3}$. If $x \in\left(B^{\sharp}\right)^{c}$ and $y \in B$, then $|y-x| \sim\left|x-x_{0}\right|$. By the canceling property of $a$, we have

$$
\begin{aligned}
\left|\left[\mathcal{L}^{i \gamma}-(-\Delta)^{i \gamma}\right](a)(x)\right| \leq & \left|\int_{B}\left[K_{\gamma}^{\mathcal{L}}(x, y)-K_{\gamma}(x, y)\right] a(y) d y\right| \\
\leq & \left|\int_{B}\left[K_{\gamma}^{\mathcal{L}}\left(x, x_{0}\right)-K_{\gamma}^{\mathcal{L}}(x, y)\right] a(y) d y\right| \\
& +\left|\int_{B}\left[K_{\gamma}(x, y)-K_{\gamma}\left(x, x_{0}\right)\right] a(y) d y\right| \\
\leq & \int_{B}|a(y)|\left[\frac{\left|y-x_{0}\right|^{\delta}}{\left|x-x_{0}\right|^{n+\delta}}+\frac{\left|y-x_{0}\right|}{\left|x-x_{0}\right|^{n+1}}\right] d y \\
\leq & C\left(\frac{r^{\delta}}{\left|x-x_{0}\right|^{n+\delta}}+\frac{r}{\left|x-x_{0}\right|^{n+1}}\right) .
\end{aligned}
$$

Then, since $r m\left(x_{0}, \mu\right)<1$, we obtain that

$$
\begin{aligned}
I_{3} & \leq C\left[\int_{\left(B^{\sharp}\right)^{c}}\left(\frac{r^{\delta}}{\left|x-x_{0}\right|^{n+\delta}}+\frac{r}{\left|x-x_{0}\right|^{n+1}}\right)^{q} d x\right]^{1 / q} \\
& \leq C\left\{\left[\int_{\left(B^{\sharp}\right) c} \frac{r^{q \delta}}{\left|x-x_{0}\right|^{q(n+\delta)}} d x\right]^{1 / q}+\left[\int_{\left(B^{\sharp}\right) c} \frac{r^{q}}{\left|x-x_{0}\right|^{q(n+1)}} d x\right]^{1 / q}\right\} \\
& \leq C r^{\delta} m\left(x_{0}, \mu\right)^{\delta+n-n / q}+r m\left(x_{0}, \mu\right)^{\delta+1-n / q} \\
& \leq C m\left(x_{0}, \mu\right)^{n-n / q} .
\end{aligned}
$$

At last, we estimate $I_{2}$. For this case, $x \in B^{\sharp} \backslash B^{*}$, then $2 r<\left|x-x_{0}\right|<2 / m\left(x_{0}, \mu\right)$ and $\mid x-$ $y|\sim| x-x_{0} \mid$. Applying (4.2) and the canceling property of $a$ again, we get

$$
\begin{aligned}
\left|\left[\mathcal{L}^{i \gamma}-(-\Delta)^{i \gamma}\right] a(x)\right| & \leq C \int_{B}\left[\left|y-x_{0}\right| m\left(x_{0}, \mu\right)\right]^{\delta^{\prime}}\left|x-x_{0}\right|^{-n}|a(y)| d y \\
& \leq C\left[m\left(x_{0}, \mu\right)\right]^{\delta^{\prime}} r^{\delta^{\prime}}\left|x-x_{0}\right|^{-n} \int_{B}|a(y)| d y \\
& \leq C\left[m\left(x_{0}, \mu\right)\right]^{\delta^{\prime}} r^{\delta^{\prime}}\left|x-x_{0}\right|^{-n} \\
& \leq C 2^{-m \delta^{\prime}}\left|x-x_{0}\right|^{-n}
\end{aligned}
$$

which implies that

$$
\begin{aligned}
I_{2} & \leq C\left[\int_{B^{\sharp} \backslash B^{*}}\left|\left[\mathcal{L}^{i \gamma}-(-\Delta)^{i \gamma}\right] a(x)\right|^{q} d x\right]^{1 / q} \\
& \leq C\left(\int_{B^{\sharp} \backslash B^{*}} 2^{-m \delta^{\prime} q}\left|x-x_{0}\right|^{-n q} d x\right)^{1 / q} \\
& \leq C m\left(x_{0}, \mu\right)^{n-n / q},
\end{aligned}
$$

where in the last inequality we have used the fact that $q<n /\left(n-\delta^{\prime}\right)$.

Finally, it follows from the estimates for $I_{i}, i=1,2,3$, that

$$
\left\|\left[\mathcal{L}^{i \gamma}-(-\Delta)^{i \gamma}\right] a\right\|_{q}^{1 /(n / q-n)} \geq m\left(x_{0}, \mu\right)^{-1}
$$


On the other hand, the $L^{q}$-boundedness of $\mathcal{L}^{i \gamma}-(-\Delta)^{i \gamma}$ gives

$$
\left\|\left[\mathcal{L}^{i \gamma}-(-\Delta)^{i \gamma}\right] a\right\|_{q} \leq\|a\|_{q}=\left(\int_{B}|a(y)|^{q} d y\right)^{1 / q} \leq r^{n / q-n} .
$$

This means that for this case, $\left(\mathcal{L}^{i \gamma}-(-\Delta)^{i \gamma}\right) a$ need not satisfy the canceling condition.

Part III: There exists a constant $C$ such that, for any $(1, \infty)$-atom, uniformly,

$$
N_{\mathcal{L}}\left(\left[\mathcal{L}^{i \gamma}-(-\Delta)^{i \gamma}\right] a\right) \leq C
$$

We write $b=1-1 / q+\varepsilon$, then $\varepsilon-b=1 / q-1$. We have proved that

$$
\left\|\left(\mathcal{L}^{i \gamma}-(-\Delta)^{i \gamma}\right) a\right\|_{q} \leq C \rho\left(x_{0}\right)^{n / q-n} \leq \rho\left(x_{0}\right)^{n(\varepsilon-b)} .
$$

Now we split: $\left\|\left|\cdot-x_{0}\right|^{n b}\left(\mathcal{L}^{i \gamma}-(-\Delta)^{i \gamma}\right) a\right\|_{q} \leq I_{1}+I_{2}$, where

$$
\left\{\begin{array}{l}
I_{1}:=\left\|\left|\cdot-x_{0}\right|^{n b}\left(\mathcal{L}^{i \gamma}-(-\Delta)^{i \gamma}\right) a\right\|_{L^{q}\left(B^{\sharp}\right)} ; \\
\left.I_{2}:=\left\|\left|\cdot-x_{0}\right|^{n b}\left(\mathcal{L}^{i \gamma}-(-\Delta)^{i \gamma}\right) a\right\|_{L^{q}\left(\left(B^{\sharp}\right) c\right.}\right) .
\end{array}\right.
$$

For $I_{1}$, because $B^{\sharp}=\left(x_{0}, 2 \rho\left(x_{0}\right)\right)$,

$$
I_{1} \leq C \rho\left(x_{0}\right)^{n b}\left[\int_{B^{\sharp}}\left|\left(\mathcal{L}^{i \gamma}-(-\Delta)^{i \gamma}\right) a(x)\right|^{q}\right]^{1 / q} \leq C \rho\left(x_{0}\right)^{n b} \rho\left(x_{0}\right)^{n(\varepsilon-b)} \leq C \rho\left(x_{0}\right)^{n \varepsilon} .
$$

For $I_{2}$, we further split $I_{2}$ into $I_{2,1}+I_{2,2}$, where

$$
\left\{\begin{array}{l}
I_{2,1}:=\left\|\left|\cdot-x_{0}\right|^{n b} \mathcal{L}^{i \gamma} a\right\|_{L^{q}\left(\left(B^{\sharp}\right)^{c}\right)} ; \\
I_{2,2}:=\left\|\left|\cdot-x_{0}\right|^{n b}(-\Delta)^{i \gamma} a\right\|_{L^{q}\left(\left(B^{\sharp}\right)^{c}\right)} .
\end{array}\right.
$$

Notice that $\varepsilon<\delta / n$ and $n b-(n+\delta)+n / q<0$. By Theorem 4.3, we have

$$
\begin{aligned}
I_{2,1} & \leq C \int_{B}|a(y)|\left[\int_{\left(B^{\sharp}\right)^{c}}\left|x-x_{0}\right|^{q n b}\left|\int_{B}\right| y-\left.x_{0}\right|^{q \delta}\left|x-x_{0}\right|^{-q(n+\delta)} d x\right]^{1 / q} d y \\
& \leq C \int_{B}|a(y)|\left|y-x_{0}\right|^{\delta}\left[\int_{\left(B^{\sharp}\right) c}\left|x-x_{0}\right|^{q n b-q(n+\delta)} d x\right]^{1 / q} d y \\
& \leq C \int_{B}|a(y)| r^{\delta} \rho\left(x_{0}\right)^{n b-(n+\delta)+n / q} d y \\
& \leq C \rho\left(x_{0}\right)^{n \varepsilon} .
\end{aligned}
$$

For $I_{2,2}$, similarly, we have

$$
\begin{aligned}
I_{2,2} & \leq C \int_{B}|a(y)|\left|x-x_{0}\right|\left(\int_{\left|x-x_{0}\right| \geq 2 \rho\left(x_{0}\right)}\left|x-x_{0}\right|^{q(n b-n-1)}\left|x-x_{0}\right|^{n-1} d\left|x-x_{0}\right|\right)^{1 / q} d y \\
& \leq C \int_{B}|a(y)| r \rho\left(x_{0}\right)^{n b-(n+1)+n / q} d y \\
& \leq C \rho\left(x_{0}\right)^{n \varepsilon}
\end{aligned}
$$


where we have used the fact that $0<\varepsilon<\min \{\delta / n, 1 / n\}$. Finally, we get

$$
\left\|\left|\cdot-x_{0}\right|^{n b}\left(\mathcal{L}^{i \gamma}-(-\Delta)^{i \gamma}\right) a\right\|_{q} \leq \rho\left(x_{0}\right)^{n \varepsilon},
$$

and, hence,

$$
\begin{aligned}
& \left\|\left(\mathcal{L}^{i \gamma}-(-\Delta)^{i \gamma}\right) a\right\|_{q}^{\varepsilon / b}\left\|\left|\cdot-x_{0}\right|^{n b}\left(\mathcal{L}^{i \gamma}-(-\Delta)^{i \gamma}\right) a\right\|_{q}^{(1-\varepsilon / b)} \\
& \quad \leq C \rho\left(x_{0}\right)^{n(\varepsilon-b) \varepsilon / b} \rho\left(x_{0}\right)^{n \varepsilon(1-\varepsilon / b)} \leq C .
\end{aligned}
$$

Finally, we have proved that, for any $(1, \infty)$-atom, $\mathcal{L}^{i \gamma} a$ is a $(1, q, \varepsilon)$-molecular or the linear combination of finite $(1, q, \varepsilon)$-moleculars.

\subsection{The $H_{\mathcal{L}}^{1}$-boundedness of Riesz transforms $R_{\mathcal{L}}$}

In this section, we prove that Riesz transforms $R_{\mathcal{L}}$ are bounded on $H_{\mathcal{L}}^{1}\left(\mathbb{R}^{n}\right)$. The Riesz transforms associated with $\mathcal{L}$ are defined as

$$
R_{\mathcal{L}}:=\nabla(-\Delta+\mu)^{-1 / 2}
$$

where $(-\Delta+\mu)^{-1 / 2}=\frac{1}{\pi} \int_{0}^{\infty} \lambda^{-1 / 2}(-\Delta+\mu+\lambda)^{-1} d \lambda$. Shen proved the following estimate of $R_{\mathcal{L}}$. Assume that $\mu$ satisfies (1.2) \& (1.3) for some $\delta>1$. Then $\nabla(-\Delta+\mu)^{-1 / 2}$ is a CalderónZygmund operator. Precisely,

$$
\nabla(-\Delta+\mu)^{-1 / 2} f(x)=\int_{\mathbb{R}^{n}} R_{\mathcal{L}}(x, y) f(y) d y,
$$

where

$$
R_{\mathcal{L}}(x, y):=\frac{1}{\pi} \int_{0}^{\infty} \lambda^{-1 / 2} \nabla_{x} \Gamma_{\mu+\lambda}(x, y) d \lambda .
$$

In [12], Shen proved the following results, see [12, (7.20), (7.26), (7.29)], respectively.

Lemma 4.5 The kernel $R_{\mathcal{L}}(\cdot, \cdot)$ satisfies the following estimates:

$$
\begin{cases}(1) & \left|R_{\mathcal{L}}(x, y)\right| \leq C e^{-c d(x, y, \mu)}|x-y|^{-n} \\ (2) & \left|R_{\mathcal{L}}(x+h, y)-R_{\mathcal{L}}(x, y)\right| \leq C(|h| /|x-y|)^{\delta-1}|x-y|^{-n} ; \\ (3) & \left|R_{\mathcal{L}}(x, y+h)-R_{\mathcal{L}}(x, y)\right| \leq C(|h| /|x-y|)^{\delta_{1}}|x-y|^{-n}, \quad \delta_{1} \in(0,1) .\end{cases}
$$

Theorem 4.6 Let $\mathcal{L}=-\Delta+\mu$ be a generalized Schrödinger operator, where $\mu \neq 0$ is a nonnegative Radon measure on $\mathbb{R}^{n}$ satisfying (1.2) \& (1.3) for some $\delta>0$. The Riesz transform $R_{\mathcal{L}}$ is bounded on $H_{\mathcal{L}}^{1}\left(\mathbb{R}^{n}\right)$.

Proof Similar to Theorem 4.4, the proof of this theorem is divided into three parts.

Part I: $\left\||\cdot|{ }^{n b} R_{\mathcal{L}} a\right\|_{q}<\infty$, uniformly. For any atom $a$ and $B^{*}=B\left(x_{0}, 2 r\right)$, we write $\left\||\cdot|{ }^{n b} R_{\mathcal{L}} a\right\|_{q} \leq I_{1}+I_{2}$, where $I_{1}:=\left\||\cdot|{ }^{n b} R_{\mathcal{L}} a\right\|_{L^{q}\left(B^{*}\right)}$ and $I_{2}:=\left\||\cdot|{ }^{n b} R_{\mathcal{L}} a\right\|_{L^{q}\left(\left(B^{*}\right) c\right.}$.

By the $L^{q}$-boundedness of $R_{\mathcal{L}}$, we have

$$
I_{1} \leq r^{n b}\left(\int_{B^{*}}\left|R_{\mathcal{L}} a(x)\right|^{q} d x\right)^{1 / q} \leq r^{n b}\|a\|_{q} \leq r^{n b}\left|B\left(x_{0}, r\right)\right|^{1 / q-1} \leq r^{n \varepsilon} .
$$


By Lemma 4.5, for any positive $N>0$,

$$
\left|R_{\mathcal{L}}(x, y)\right| \leq C|x-y|^{-n-N}[m(y, \mu)]^{-N}
$$

On the other hand, for $y \in B$ and $x \in\left(B^{*}\right)^{c},|x-y| \geq\left|x-x_{0}\right| / 2$. We can obtain that

$$
\begin{aligned}
I_{2} \leq & C \int_{B}|a(y)|\left(\int_{\left(B^{*}\right)^{c}}|x|^{q n b}\left|R_{\mathcal{L}}(x, y)\right|^{q} d x\right)^{1 / q} d y \\
\leq & C \int_{B}|a(y)| \frac{1}{m(y, \mu)^{N}}\left(\int_{\left(B^{*}\right)^{c}}|x|^{q n b} \frac{1}{|x-y|^{(n+N) q}} d x\right)^{1 / q} d y \\
\leq & C\left\{\int_{B}|a(y)| \frac{\left|x_{0}\right|^{n b}}{m(y, \mu)^{N}}\left(\int_{\left(B^{*}\right)^{c}} \frac{d x}{\left|x-x_{0}\right|^{(n+N) q}}\right)^{1 / q} d y\right. \\
& \left.+\int_{B}|a(y)| \frac{1}{m(y, \mu)^{N}}\left(\int_{\left(B^{*}\right)^{c}} \frac{\left|x-x_{0}\right|^{q n b}}{\left|x-x_{0}\right|^{(n+N) q}} d x\right)^{1 / q} d y\right\} \\
\leq & C \int_{B} \frac{|a(y)|}{m(y, \mu)^{N}}\left\{r^{n \epsilon-N}+\left|x_{0}\right|^{-n-N+n / q}\right\} d y .
\end{aligned}
$$

Because $y \in B\left(x_{0}, r\right)$,

$$
m(y, \mu) \geq \frac{C m\left(x_{0}, \mu\right)}{\left\{1+\left|x-x_{0}\right| m\left(x_{0}, \mu\right)\right\}^{k_{0} /\left(k_{0}+1\right)}},
$$

which implies that $\left\||\cdot|^{n b} a\right\|_{L^{q}\left(\left(B^{*}\right)^{c}\right)}<\infty$.

Part II: $N_{\mathcal{L}}\left(R_{\mathcal{L}}(a)\right) \leq C$. We divide the proof into two cases.

Case1: $r \geq 1 / m\left(x_{0}, \mu\right)$. By the boundedness of the Riesz transform $R_{\mathcal{L}}$, we have

$$
\begin{aligned}
\left\|R_{\mathcal{L}} a\right\|_{q} & \leq C\left\{\left\|R_{\mathcal{L}} a\right\|_{q}+\left\|\chi_{\left(B^{*}\right)^{c}} R_{\mathcal{L}} a\right\|_{q}\right\} \\
& \leq C\left\{\|a\|_{q}+\left\|\chi_{\left(B^{*}\right)^{c}} R_{\mathcal{L}} a\right\|_{q}\right\} \\
& \leq C\left\{\left|B\left(x_{0}, r\right)\right|^{1 / q-1}+\int_{B}|a(y)|\left(\int_{\left(B^{\sharp}\right) c}\left|R_{\mathcal{L}}(x, y)\right|^{q} d x\right)^{1 / q} d y\right\} .
\end{aligned}
$$

By Lemma 4.5, we can get

$$
\begin{aligned}
\int_{B}|a(y)|\left(\int_{\left(B^{\sharp}\right) c}\left|R_{\mathcal{L}}(x, y)\right|^{q} d x\right)^{1 / q} d y & \leq C \int_{B}|a(y)|\left(\int_{2 r}^{\infty} s^{n-q n-1} d s\right)^{1 / q} d y \\
& \leq C \int_{B}|a(y)| d y \times r^{n / q-n} \\
& \leq C \rho\left(x_{0}\right)^{n / q-n},
\end{aligned}
$$

which means that $\left\|R_{\mathcal{L}} a\right\|_{q}^{1 /(n / q-n)} \geq \rho\left(x_{0}\right)$, i.e., $R_{\mathcal{L}} a$ does not need the canceling condition for this case. Now we split $\left\|\left|\cdot-x_{0}\right|^{n b} R_{\mathcal{L}} a\right\|_{q} \leq I_{1}+I_{2}$, where

$$
\left\{\begin{array}{l}
I_{1}:=\left(\int_{B^{*}}\left|x-x_{0}\right|^{q n b}\left|R_{\mathcal{L}} a(x)\right|^{q} d x\right)^{1 / q} ; \\
I_{2}:=\left(\int_{\left(B^{*}\right)^{c}}\left|x-x_{0}\right|^{q n b}\left|R_{\mathcal{L}} a(x)\right|^{q} d x\right)^{1 / q} .
\end{array}\right.
$$


It is easy to see that

$$
I_{1} \leq r^{n b}\left(\int_{B^{*}}\left|R_{\mathcal{L}} a(x)\right|^{q} d x\right)^{1 / q} \leq r^{n b}\left\|R_{\mathcal{L}} a\right\|_{q} \leq r^{n b}\|a\|_{q} \leq r^{n \varepsilon} .
$$

For $I_{2}$, by Minkowski's inequality,

$$
\begin{aligned}
I_{2} & \leq C \int_{B}|a(y)| m(y, \mu)^{-N}\left(\int_{B^{*}}\left|x-x_{0}\right|^{q n b-q(n+N)} d x\right)^{1 / q} d y \\
& \leq C \int_{B}|a(y)| m(y, \mu)^{-N} r^{n b+n / q-n-N} d y \\
& \leq C \int_{B}|a(y)|\left\{m\left(x_{0}, \mu\right)^{-1}+\left|y-x_{0}\right|\right\}^{N} r^{n b+n / q-n-N} d y \\
& \leq C \int_{B}|a(y)| r^{N} r^{n b+n / q-n-N} d y \\
& \leq C|B|^{(\varepsilon-b) \varepsilon / b}|B|^{\varepsilon(1-\varepsilon / b)}
\end{aligned}
$$

which gives $N_{\mathcal{L}}\left(R_{\mathcal{L}} a\right)=\left\|R_{\mathcal{L}} a\right\|_{q}^{\varepsilon / b}\left\|\left|\cdot-x_{0}\right|^{n b} R_{\mathcal{L}} a\right\|_{q}^{1-\varepsilon / b} \leq C$.

Case 2: $r \leq \rho\left(x_{0}\right)$. Let $B^{\sharp}=B\left(x_{0}, 2 \rho\left(x_{0}\right)\right)$ and $B^{*}=B\left(x_{0}, 2 r\right)$. So $R_{\mathcal{L}} a=R_{0} a+\left(R_{\mathcal{L}}-R_{0}\right) a$, where $R_{0}:=\nabla(-\Delta)^{-1 / 2}$. For any $a$ with the canceling condition, $R_{0} a$ is a molecular. We only need to deal with $\left(R_{\mathcal{L}}-R_{0}\right) a$. Split $\left\|\left(R_{\mathcal{L}}-R_{0}\right) a\right\|_{q} \leq I_{1}+I_{2}+I_{3}$, where

$$
\left\{\begin{array}{l}
I_{1}:=\left(\int_{B^{*}}\left|\left(R_{\mathcal{L}}-R_{0}\right) a(x)\right|^{q} d x\right)^{1 / q} ; \\
I_{2}:=\left(\int_{B^{\sharp} \backslash B^{*}}\left|\left(R_{\mathcal{L}}-R_{0}\right) a(x)\right|^{q} d x\right)^{1 / q} ; \\
I_{3}:=\left(\int_{\left(B^{\sharp}\right) c}\left|\left(R_{\mathcal{L}}-R_{0}\right) a(x)\right|^{q} d x\right)^{1 / q} .
\end{array}\right.
$$

We first estimate $I_{3}$. For $x \in\left(B^{\sharp}\right)^{c}$ and $y \in B,|x-y| \sim\left|x-x_{0}\right|$. Denote by $R_{0}(\cdot, \cdot)$ the kernel of $\nabla(-\Delta)^{-1 / 2}$. We can get

$$
\begin{aligned}
& \left|\left(R_{\mathcal{L}}-R_{0}\right)(a)(x)\right| \\
& \quad \leq C\left\{\int_{B}\left|R_{\mathcal{L}}(x, y)-R_{\mathcal{L}}\left(x, x_{0}\right)\right||a(y)| d y+\int_{B}\left|R_{0}(x, y)-R_{0}\left(x, x_{0}\right)\right||a(y)| d y\right\} \\
& \quad \leq C\|a\|_{\infty}\left(\int_{B} \frac{\left|y-x_{0}\right|^{\delta}}{\left|x-x_{0}\right|^{n+\delta}} d y+\int_{B} \frac{\left|y-x_{0}\right|}{\left|x-x_{0}\right|^{n+1}} d y\right) \\
& \quad \leq C\|a\|_{\infty}|B|\left(\frac{r^{\delta}}{\left|x-x_{0}\right|^{n+\delta}}+\frac{r}{\left|x-x_{0}\right|^{n+1}}\right) .
\end{aligned}
$$

It follows from the above estimate that

$$
\begin{aligned}
I_{3} & \leq C\left\{\left(\int_{\left(B^{\sharp}\right)} \frac{r^{q \delta}}{\left|x-x_{0}\right|^{q(n+\delta)}} d x\right)^{1 / q}+\left(\int_{\left(B^{\sharp}\right)^{c}} \frac{r^{q}}{\left|x-x_{0}\right|^{q(n+1)}} d x\right)^{1 / q}\right\} \\
& \leq C\left\{r^{\delta} m\left(x_{0}, \mu\right)^{n+\delta-n / q}+r\left[m\left(x_{0}, \mu\right)\right]^{n+1-n / q}\right\} \\
& \leq C\left[m\left(x_{0}, \mu\right)\right]^{n-n / q} .
\end{aligned}
$$


For the estimates of $I_{1} \& I_{2}$, we need the following estimate:

$$
\left|R_{\mathcal{L}}(y, x)-R_{0}(y, x)\right| \leq C\left\{\frac{1}{r^{n-1}} \int_{B(y, r)} \frac{d \mu(z)}{|z-y|^{n-1}}+\frac{(r m(x, \mu))^{\delta}}{r^{n}}\right\} .
$$

For $r=|x-y| / 2$,

$$
\left|R_{\mathcal{L}}(y, x)-R_{0}(y, x)\right| \leq C\left\{\frac{1}{|x-y|^{n-1}} \int_{B(y,|x-y| / 2)} \frac{d \mu(z)}{|z-y|^{n-1}}+\frac{(|x-y| m(x, \mu))^{\delta}}{|x-y|^{n}}\right\} .
$$

We get $I_{1} \leq \int_{B}|a(y)| A_{1}(y) d y$, where

$$
A_{1}:=\left\{\int_{B^{*}}\left|R_{\mathcal{L}}(x, y)-R_{0}(x, y)\right|^{q} d x\right\}^{1 / q} .
$$

Due to (4.4), we further obtain $A_{1} \leq U_{1}+U_{2}$, where

$$
\left\{\begin{array}{l}
U_{1}:=\left\{\int_{B^{\sharp}}|x-y|^{q(1-n)}\left(\int_{B(x,|x-y| / 2)}|z-x|^{1-n} d \mu(z)\right)^{q} d x\right\}^{1 / q} ; \\
U_{2}:=\left\{\int_{B^{\sharp}}(|x-y| m(y, \mu))^{q \delta}|x-y|^{-q n} d x\right\}^{1 / q} .
\end{array}\right.
$$

For $U_{2}$, if $y \in B$, then $\left|y-x_{0}\right|<r<2 \rho\left(x_{0}\right)$ and $m(y, \mu) \sim m\left(x_{0}, \mu\right)$. On the other hand, because $x \in B^{\sharp}$, then $|x-y|<3 / m\left(x_{0}, \mu\right)$. We can get

$$
U_{2} \leq C m\left(x_{0}, \mu\right)^{\delta}\left(\int_{B^{\sharp}}|x-y|^{q \delta-q n} d x\right)^{1 / q} \leq C m\left(x_{0}, \mu\right)^{n-n / q} .
$$

Now we estimate the term $U_{1}$. Let $T_{j}=B\left(y, 2^{j+2} / m\left(x_{0}, \mu\right)\right)$. If $y \in B$ and $x \in B^{\sharp}$, by the triangle inequality, it is easy to see that $B^{\sharp} \subset B\left(y, 4 / m\left(x_{0}, \mu\right)\right)$. Also, for $x \in T_{j+1} \backslash T_{j},|x-y| \geq$ $2^{j+2} / m\left(x_{0}, \mu\right)$. On the other hand, $B(x,|x-y| / 2) \subset B(y, 3|x-y| / 2)$. Then

$$
\begin{aligned}
U_{1} & \leq C \sum_{j=-\infty}^{0}\left(\int_{T_{j+1} \backslash T_{j}} \frac{1}{|x-y|^{q(n-1)}}\left(\int_{B(x,|x-y| / 2)} \frac{d \mu(z)}{|z-x|^{n-1}}\right)^{q} d x\right)^{1 / q} \\
& \leq C \sum_{j=-\infty}^{0}\left[\frac{m\left(x_{0}, \mu\right)}{2^{j}}\right]^{n-1}\left(\int_{T_{j+1} \backslash T_{j}}\left(\int_{B(y,|x-y| / 2)} \frac{d \mu(z)}{|z-x|^{n-1}}\right)^{q} d x\right)^{1 / q} \\
& \leq C \sum_{j=-\infty}^{0}\left[\frac{m\left(x_{0}, \mu\right)}{2^{j}}\right]^{n-1}\left(\int_{T_{j+1} \backslash T_{j}}\left(\int_{B\left(y, 2^{j+2} / m\left(x_{0}, \mu\right)\right)} \frac{d \mu(z)}{|z-x|^{n-1}}\right)^{q} d x\right)^{1 / q} \\
& \leq C \sum_{j=-\infty}^{0}\left[\frac{m\left(x_{0}, \mu\right)}{2^{j}}\right]^{n-1} \frac{\mu\left(3 T_{j+1}\right)}{2^{(j+2)(n-n / q-1)}}\left[m\left(x_{0}, \mu\right)\right]^{n-n / q-1} .
\end{aligned}
$$

Notice that

$$
\mu\left(T_{j+1}\right)=\mu\left(B\left(y, 2^{j+2} / m\left(x_{0}, \mu\right)\right)\right) \leq\left(2^{j+2}\right)^{n-2+\delta} m\left(x_{0}, \mu\right)^{2-n} .
$$

A direct computation gives

$$
U_{1} \leq C \sum_{j=-\infty}^{0}\left[m\left(x_{0}, \mu\right)\right]^{n-n / q} 2^{j\left(n-2+\delta^{\prime}\right)} 2^{-j(2 n-1 / q-2)}
$$




$$
\begin{aligned}
& \leq C\left[m\left(x_{0}, \mu\right)\right]^{n-n / q} \sum_{j=-\infty}^{0} 2^{j\left(n-2+\delta^{\prime}-2 n+n / q+2\right)} \\
& \leq C\left[m\left(x_{0}, \mu\right)\right]^{n-n / q},
\end{aligned}
$$

which implies that

$$
I_{1} \leq C \int_{B}|a(y)| A_{1}(y) d y \leq C\|a\|_{1}\left[m\left(x_{0}, \mu\right)\right]^{n-n / q} \leq C\left[m\left(x_{0}, \mu\right)\right]^{n-n / q} .
$$

The estimate for $I_{2}$ is similar. Then we obtain $\left\|\left(R_{\mathcal{L}}-R_{0}\right) a\right\|_{q}^{1 /\{n / q-n\}} \geq C / m\left(x_{0}, \mu\right)$, which means $\left(R_{\mathcal{L}}-R_{0}\right)$ a does not need the canceling condition. What is left to prove is the norm $\left\|\left|\cdot-x_{0}\right|^{n b}\left(R_{\mathcal{L}}-R_{0}\right) a\right\|_{q}$. We write $\left\|\left|\cdot-x_{0}\right|^{n b}\left(R_{\mathcal{L}}-R_{0}\right) a\right\|_{q} \leq E_{1}+E_{2}$, where

$$
\left\{\begin{array}{l}
E_{1}:=\left(\int_{B^{\sharp}}\left|x-x_{0}\right|^{q n b}\left|\left(R_{\mathcal{L}}-R_{0}\right) a(x)\right|^{q} d x\right)^{1 / q} ; \\
E_{2}:=\left(\int_{\left(B^{\sharp}\right) c}\left|x-x_{0}\right|^{q n b}\left|\left(R_{\mathcal{L}}-R_{0}\right) a(x)\right|^{q} d x\right)^{1 / q} .
\end{array}\right.
$$

By the $L^{p}$-boundedness of $R_{\mathcal{L}}$ and $R_{0}$, we get

$$
E_{1} \leq C m\left(x_{0}, \mu\right)^{-n b}\left\|\left(R_{\mathcal{L}}-R_{0}\right) a\right\|_{q} \leq C m\left(x_{0}, \mu\right)^{-n b}\|a\|_{q} \leq C m\left(x_{0}, \mu\right)^{-n \varepsilon} .
$$

For the term $E_{2}$, we have $E_{2} \leq E_{2,1}+E_{2,2}$, where

$$
\left\{\begin{array}{l}
E_{2,1}:=\left(\int_{\left(B^{\sharp}\right)^{c}}\left|x-x_{0}\right|^{q n b}\left|R_{\mathcal{L}} a(x)\right|^{q} d x\right)^{1 / q} ; \\
E_{2,2}:=\left(\int_{\left(B^{\sharp}\right)^{c}}\left|x-x_{0}\right|^{q n b}\left|R_{0} a(x)\right|^{q} d x\right)^{1 / q} .
\end{array}\right.
$$

A direct computation gives

$$
\begin{aligned}
E_{2,1} & \leq C \int_{B}|a(y)| d y\left(\int_{\left(B^{\sharp}\right) c}\left|x-x_{0}\right|^{q n b}\left|R_{\mathcal{L}}(x, y)-R_{\mathcal{L}}\left(x, x_{0}\right)\right|^{q} d x\right)^{1 / q} \\
& \leq C \int_{B}|a(y)|\left|y-x_{0}\right|^{\delta}\left(\int_{\left(B^{\sharp}\right) c} \frac{\left|x-x_{0}\right|^{q n b}}{\left|x-x_{0}\right|^{(n+\delta) q}} d x\right)^{1 / q} d y \leq C m\left(x_{0}, \mu\right)^{-n \varepsilon} .
\end{aligned}
$$

For $E_{2,2}$, because $R_{0}$ is a Calderón-Zygmund operator, the kernel $K_{0}(\cdot, \cdot)$ satisfies

$$
\left|R_{0}(x, y)-R_{0}\left(x, x_{0}\right)\right| \leq C\left|y-x_{0}\right|\left|x-x_{0}\right|^{-n-1} .
$$

We can get

$$
\begin{aligned}
E_{2,2} & \leq C \int_{B}|a(y)|\left(\int_{\left(B^{\sharp}\right) c}\left|x-x_{0}\right|^{q(n b-n)} d x\right)^{1 / q} d y \\
& \leq C r^{n+1-n / q}\left(\int_{2 / m\left(x_{0}, \mu\right)}^{\infty} s^{-(q-1) n-q+n b q-1} d s\right)^{1 / q} \leq C m\left(x_{0}, \mu\right)^{-n \varepsilon} .
\end{aligned}
$$

Finally, we obtain that

$$
N_{L}\left(\left(R_{\mathcal{L}}-R_{0}\right) a\right) \leq C m\left(x_{0}, \mu\right)^{(n / q-n)(\varepsilon / b)} m\left(x_{0}, \mu\right)^{n \varepsilon(\varepsilon / b-1)} \leq C .
$$




\section{Acknowledgements}

Some part of this manuscript was completed when the second author was visiting Mudanjiang Normal University. The authors would like to express their deepest thanks to Professor Pu Zhang for his discussion on this topic.

\section{Funding}

Project supported by the National Natural Science Foundation of China under grants No. 11871293 \& No. 11571217 Shandong Natural Science Foundation of China (No. ZR2017JL008, No. ZR2016AM05); University Science and Technology Projects of Shandong Province (No. J15LI15).

\section{Availability of data and materials}

We declare that the materials described in the manuscript, including all relevant raw data, will be freely available to any scientist wishing to use them for non-commercial purposes, without breaching participant confidentiality.

\section{Competing interests}

The authors declare that they have no competing interests.

\section{Authors' contributions}

Both authors conceived of the study, participated in its design and coordination, drafted the manuscript, and participated in the sequence alignment. All authors read and approved the final manuscript.

\section{Publisher's Note}

Springer Nature remains neutral with regard to jurisdictional claims in published maps and institutional affiliations.

Received: 26 December 2018 Accepted: 4 June 2019 Published online: 14 June 2019

\section{References}

1. Alvarez, J., Milman, M.: H $H^{p}$ continuty properties of Calderón-Zygmund operators. J. Math. Anal. Appl. 118, 65-79 (1986)

2. Cao, J., Yang, Da.: Hardy spaces $H_{L}^{p}\left(\mathbb{R}^{n}\right)$ associated with operators satisfying $k$-Davies-Gaffney estimates. Sci. China Math. 55, 1403-1440 (2012)

3. Dong, J., Huang, J., Liu, H.: Boundedness of singular integrals on Hardy type spaces associated with Schrödinger operators. J. Funct. Spaces 2015, Article ID 409215 (2015)

4. Duong, X., Yan, L.: Duality of Hardy and BMO spaces associated with operators with heat kernel bounds. J. Am. Math. Soc. 18, 943-973 (2005)

5. Dziubański, J.: Note on $H^{1}$ spaces related to degenerate Schrödinger operators. III. J. Math. 49, 1271-1297 (2005)

6. Dziubański, J., Zienkiewicz, J.: Hardy space $H^{1}$ associated to Schrödinger operator with potential satisfying reverse Hölder inequality. Rev. Mat. Iberoam. 15, 279-296 (1999)

7. Hofmann, S., Lu, G., Mitrea, D., Mitrea, M., Yan, L.: Hardy spaces associated to non-negative self-adjoint operators satisfying Davies-Gaffney estimates. Mem. Am. Math. Soc. 214 no. 1007 (2011)

8. Jiang, R., Yang, Da.: Orlicz-Hardy spaces associated with operators. Sci. China Ser. A 52, 1042-1080 (2009)

9. Jiang, R., Yang, Da.: Orlicz-Hardy spaces associated with operators satisfying Davies-Gaffney estimates. Commun. Contemp. Math. 13, 331-373 (2011)

10. Lin, C., Liu, H., Liu, Y.: Hardy spaces associated with Schrödinger operators on the Heisenberg group. arXiv: 1106.4960 [math.AP]

11. Liu, Y., Qi, S.: Endpoint estimates of Riesz transforms associated with generalized Schrödinger operators. Can. Math. Bull. 61, 787-801 (2018)

12. Shen, Z:: On fundamental solutions of generalized Schrödinger operators. J. Funct. Anal. 167, 521-564 (1999)

13. Song, L., Yan, L.: Riesz transforms associated to Schrödinger operators on weighted Hardy spaces. J. Funct. Anal. 259, 1466-1490 (2010)

14. Stein, E.M.: Harmonic Analysis: Real Variable Methods, Orthogonality and Oscillatory Integrals. Princeton Math. Serises, vol. 43. Princeton University Press, Princeton (1993)

15. Wu, L., Yan, L.: Heat kernel, upper bounds and Hardy spaces associated to the generalized Schrödinger operators. J. Funct. Anal. 270, 3709-3749 (2016)

\section{Submit your manuscript to a SpringerOpen ${ }^{\circ}$ journal and benefit from:}

- Convenient online submission

- Rigorous peer review

- Open access: articles freely available online

- High visibility within the field

Retaining the copyright to your article

Submit your next manuscript at $>$ springeropen.com 